INPLASY

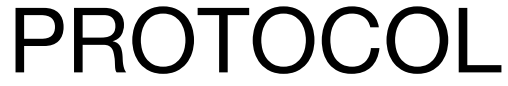

To cite: Wang et al.

Acupuncture for knee osteoarthritis: a systematic review and Meta-Analysis. Inplasy protocol 2020110086. doi:

10.37766/inplasy2020.11.0086

Received: 19 November 2020

Published: 20 November 2020

Corresponding author:

Kai Wang

947704057@qq.com

Author Affiliation:

Acupuncture and Tuina

School/the Third Teaching

Hospital, Chengdu University

of Traditional Chinese

Medicine

Support: Number $\mathbf{8 2 0 7 4 5 5 8 .}$

Review Stage at time of this submission: The review has not yet started.

Conflicts of interest:

None.

\section{Acupuncture for knee osteoarthritis: a systematic review and Meta-Analysis}

Wang, K1; Song, W2; Chen, L3; Liao, C4; Yao, J5; Yang, Y6; Zhou, S7.

Review question / Objective: Whether acupuncture is effective and safe for knee osteoarthritis.

Condition being studied: Osteoarthritis is the leading cause of disability among older adults. The joint most commonly affected by osteoarthritis is the knee. The prevalence, disability, and associated costs of knee osteoarthritis are expected to steadily increase because of aging in the population. Nonsteroidal anti-inflammatory drugs (NSAIDs) and acetaminophen are the most commonly used pharmacologic agents for treating knee osteoarthritis. Many NSAIDs are associated with considerable side effects. Acetaminophen is modestly less effective than NSAIDs and that the clinical significance of acetaminophen is questionable because it results in only a 5\% greater improvement from baseline in pain than does placebo in the short term.

INPLASY registration number: This protocol was registered with the International Platform of Registered Systematic Review and Meta-Analysis Protocols (INPLASY) on 20 November 2020 and was last updated on 20 November 2020 (registration number INPLASY2020110086).

\section{INTRODUCTION}

Review question / Objective: Whether acupuncture is effective and safe for knee osteoarthritis.
Condition being studied: Osteoarthritis is the leading cause of disability among older adults. The joint most commonly affected by osteoarthritis is the knee. The prevalence, disability, and associated costs of knee osteoarthritis are expected to 
steadily increase because of aging in the population. Nonsteroidal anti-inflammatory drugs (NSAIDs) and acetaminophen are the most commonly used pharmacologic agents for treating knee osteoarthritis. Many NSAIDs are associated with considerable side effects. Acetaminophen is modestly less effective than NSAIDs and that the clinical significance of acetaminophen is questionable because it results in only a $5 \%$ greater improvement from baseline in pain than does placebo in the short term.

\section{METHODS}

Participant or population: Osteoarthritis of the knee patients.

\section{Intervention: Acupuncture.}

Comparator: 1. No treatment or waiting list-control during the trial period. 2. Sham acupuncture (intervention resembling verum acupuncture treatment but using superficial needle insertion, needle insertion at non-acupuncture points or at points not indicated for the condition under study and 'placebo' needles that seem to be inserted into skin but actually are not etc). Trials that have intervention groups comparing either acupuncture alone with sham intervention alone or acupuncture plus one or more therapies with sham intervention plus the same therapies also will be included. 3. Pharmacological treatment that is given as a control during a comparable time as application of acupuncture treatment.

Study designs to be included: Randomized, controlled trials.

Eligibility criteria: All RCTs that compared needle acupuncture with a sham, usual care, or waiting list control group for patients of knee osteoarthritis will be included.

Information sources: We will search the Cochrane Central Register of Controlled Trials, MEDLINE, EMBASE databases as well as four Chinese data- bases (Chinese Biomedical Literature Database, China
National Knowledge Infrastructure, Chinese Science and Technology Periodical Database, and Wanfang Data- base) from inception to 30 October 2020. No language restriction will be applied.

Main outcome(s): The main outcome was the response rate.

Quality assessment / Risk of bias analysis: Reporting bias will be explored by constructing funnel plots and performing Egger's test, if there are at least 10 trials included in meta-analysis.

Strategy of data synthesis: The synthesis will be done by generating a forest plot for meta-regression. This plot does not contain a summary measure given by a prism below the single studies, but by a prism shown for each single study that shows the aggregated effect for the specific type of study (depending on the covariates of the meta-regression). If the heterogeneity test indicates there is no substantial heterogeneity between studies, the MantelHaenszel method implemented by the rma. $\mathrm{mh}$ () function will be fitted for calculating pooled estimates, $95 \% \mathrm{Cls}$ and combined $\mathrm{p}$ values. The significance of the $p$ value represents the strength of evidence against the null hypothesis of no intervention effect.

Subgroup analysis: Subgroup analysis will be performed according to the primary and secondary objectives. To detect possible heterogeneity of the results, subgroup analysis will be conducted for both the primary outcome and secondary outcomes at the end of the treatment session and the end of the follow-up period.

Sensibility analysis: In order to obtain a stable conclusion, a sensitivity analysis will be conducted to remove effects of trials with small sample size and remove studies rated as high risk of bias based on accounting of methodological quality. These steps will be crucial to ensure the accuracy and depth of inferences from results.

Country(ies) involved: China. 
Keywords: osteoarthritis of the knee; acupuncture; meta-analysis.

Contributions of each author:

Author 1 - Kai Wang.

Author 2 - Wei Song.

Author 3 - Li Chen.

Author 4 - Chenxi Liao.

Author 5 - Junpeng Yao.

Author 6 - Yuqing Yang.

Author 7 - Siyuan Zhou. 